\title{
Efficacy and Toxicity of Panitumumab After Progression on Cetuximab and Predictive Value of MiR-31-5p in Metastatic Wild-type KRAS Colorectal Cancer Patients
}

\author{
IGOR KISS ${ }^{1}$, JITKA MLCOCHOVA $^{2}$, ZBYNEK BORTLICEK $^{3}$, ALEXANDR POPRACH ${ }^{1}$, \\ JIRI DRABEK ${ }^{4}$, PETRA VYCHYTILOVA-FALTEJSKOVA ${ }^{1,2}$, MAREK SVOBODA $^{1,2}$, \\ TOMAS BUCHLER ${ }^{5}$, STANISLAV BATKO ${ }^{6}$, ALES RYSKA ${ }^{7}$, MARIAN HAJDUCH ${ }^{4}$ and ONDREJ SLABY ${ }^{1,2}$ \\ ${ }^{1}$ Department of Comprehensive Cancer Care, Masaryk Memorial Cancer Institute, Brno, Czech Republic; \\ ${ }^{2}$ Central European Institute of Technology, Masaryk University, Brno, Czech Republic; \\ ${ }^{3}$ Institute of Biostatistics and Analyses, Faculty of Medicine, Masaryk University, Brno, Czech Republic; \\ ${ }^{4}$ Institute of Molecular and Translational Medicine, Faculty of Medicine and Dentistry, \\ Palacky University Olomouc, Olomouc, Czech Republic; \\ ${ }^{5}$ Department of Oncology, Thomayer Hospital and Charles University First \\ Faculty of Medicine, Prague, Czech Republic; \\ ${ }^{6}$ Department of Oncology, Motol University Hospital and Charles University \\ Second Faculty of Medicine, Prague, Czech Republic; \\ ${ }^{7}$ The Fingerland Department of Pathology, Charles University Medical \\ Faculty Hospital, Hradec Kralove, Czech Republic
}

\begin{abstract}
Background: In metastatic colorectal cancer $(m C R C)$, panitumumab is generally considered to be ineffective after the progression on cetuximab therapy. However, few studies have demonstrated that a small subset of $m C R C$ patients may benefit from panitumumab in this setting. Patients and Methods: In our study, wild-type KRAS $m C R C$ patients, enrolled into the nationwide Czech registry CORECT between January 2007 and December 2012, were screened for panitumumab therapy after progression on cetuximab. Results: We identified $26 \mathrm{mCRC}$ in the registry with well documented progression on cetuximab in combination with irinotecan-based chemotherapy (FOLFIRI or irinotecan alone) who received panitumumab monotherapy. Partial response (PR) was achieved in $3(11.5 \%)$ patients and stable disease (SD) in 7 (26.9\%) patients after 8 weeks of
\end{abstract}

Correspondence to: Igor Kiss, MD, Ph.D., Department of Comprehensive Cancer Care, Masaryk Memorial Cancer Institute, Zluty kopec 7, 65653 Brno, Czech Republic. Tel: +420 543131111, e-mail: kiss@mou.cz and Assoc. Prof. Ondrej Slaby, PhD, Central European Institute of Technology (CEITEC), Masaryk University, University Campus Bohunice, Building A35, Room 217, Kamenice 5, 62500 Brno, Czech Republic. Tel: +420 776494155, e-mail: on.slaby@gmail.com

Key Words: Metastatic colorectal cancer, cetuximab, panitumumab, $K R A S$, microRNA, miR-31-5p. therapy. Thirteen (50.0\%) patients had evidence of progressive disease (PD) and in $3(11.5 \%)$ cases response was not available. Furthermore, we confirmed that higher expression levels of newly described biomarker, miR-31-5p, in tumor are significantly associated with shorter progression-free survival $(P F S)$ in patients treated with cetuximab $(p=0.038)$; however, we did not observe association between miR-31-5p and response to panitumumab in $m C R C$ patients after progression on cetuximab. Conclusion: It remains possible that a subset of $m C R C$ patients may benefit from panitumumab after progression on cetuximab.

Epidermal growth factor receptor (EGFR) inhibition is routinely used in the treatment of metastatic colorectal cancer (mCRC). Two EGFR antagonists, cetuximab and panitumumab, were FDA-approved for the treatment of mCRC in 2004 and 2006, respectively. Cetuximab is a chimeric monoclonal antibody with approximately $30 \%$ murine protein that targets the ligand-binding domain of EGFR, whilst panitumumab is a fully human monoclonal antibody (1). Both antibodies promote receptor internalization and prevent EGFR auto-phosphorylation by binding to the extracellular domain and, thus, inhibiting activation of the downstream signaling pathways MAPK and PI3K (2). Each antibody has been approved for the treatment of mCRC based on various parameters, including quality of life, progression-free survival (PFS) and overall survival 


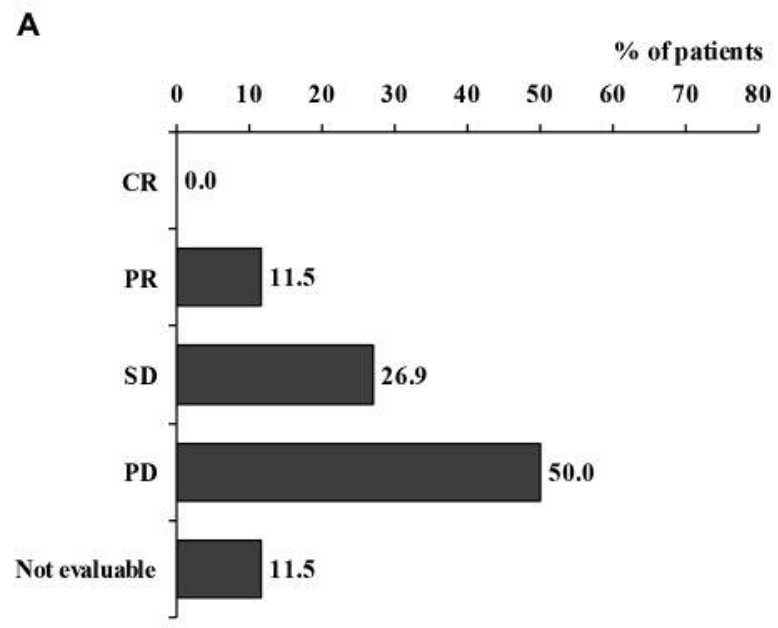

B

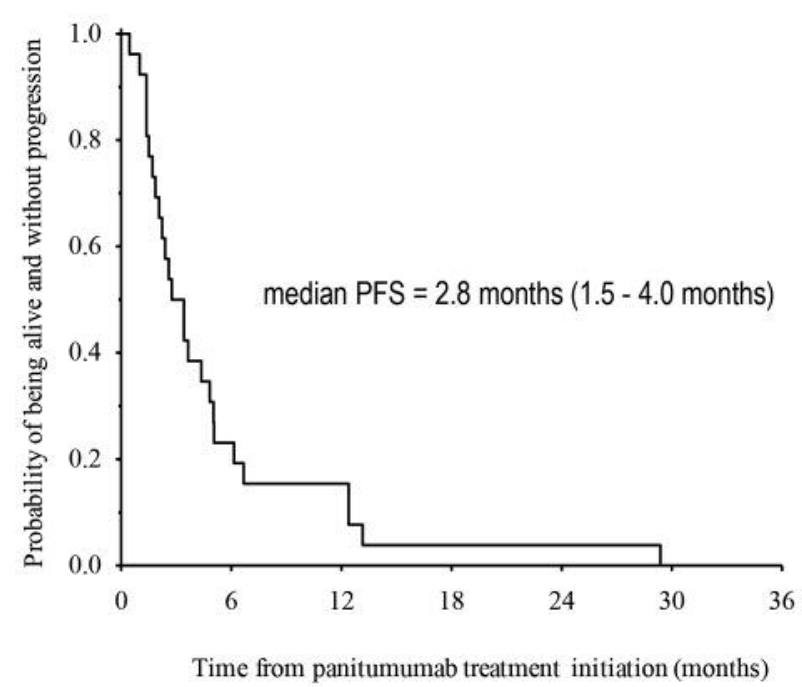

Figure 1. Treatment response $(A)$ and progression-free survival (PFS) with panitumumab $(B)$ in metastatic colorectal cancer patients after progression on cetuximab. PR, Partial response; $C R$, complete response; $S D$, stable disease; $P D$, progressive disease.

(OS), when used individually or in combination with chemotherapy (1). Although the two agents have never been directly compared in a randomized clinical trial, they produce similar response rates when used alone, as well as in combination with cytotoxic agents $(1,2)$. The rates of severe hypersensitivity reactions are somewhat increased with cetuximab (3\%) compared to panitumumab (1\%) (1).

While a series of phase III clinical trials (e.g., FIRE-3 AIO KRK-0306, CALGB/SWOG 80405) were performed to find optimal sequence of the monoclonal antibody-based treatments in wild-type $R A S$ mCRC directly comparing the addition of bevacizumab, humanized monoclonal antibody that specifically targets vascular endothelial growth factor-A (VEGF-A) versus cetuximab or panitumumab combined to FOLFOX/FOLFIRI $(3,4)$, there are only limited data describing the efficacy and toxicity of panitumumab in mCRC patients who have developed disease progression (DP) on cetuximab (5-8).

In the Czech Republic, for a reimbursement of treatment with anti-EGFR monoclonal antibodies, patient registration into the registry called CORECT, which is operated by Czech Oncological Society, is requested (9). Clinical data in this registry are updated twice a year and contain information about the effectiveness and toxicity of the anti-EGFR therapy in the individual patients. Existence of this nationwide largescale registry enables a great possibility to identify a case series of mCRC patients who received the rare treatment option based on panitumumab after progression on cetuximab and evaluate efficacy and toxicity of the treatment. As miR$31-5 p$ was recently described to be a significant predictive biomarker to anti-EGFR therapy in wild-type RAS mCRC (10-
12), we decided to further validate the predictive value of this newly described biomarker in this case series.

\section{Patients and Methods}

Metastatic CRC patients enrolled into the CORECT registry within the period of January 2007 to December 2012 were screened for well documented progression on the cetuximab in combination with irinotecan-based chemotherapy (FOLFIRI or irinotecan alone) and consequent panitumumab monotherapy. The medical records were retrieved for previous therapy, toxicity and response assessment. A response was assessed by use of the Response Evaluation Criteria in Solid Tumors (RECIST) criteria for the evaluation of measurable lesions. Stable disease (SD) was defined as stabilization for at least 8 weeks.

Further, total RNA was extracted from formalin-fixed, paraffinembedded tumor tissue samples of identified patients and miR-31-5p was quantified using the real-time polymerase chain reaction (PCR) method as we described before (12). The study was approved by the local Ethics Committee at Masaryk Memorial Cancer Institute.

PFS was defined as the time from cetuximab or panitumumab treatment initiation to the date of first documented progression or death due to any cause. Patients who had not progressed or died were censored at the date of last follow-up. PFS was estimated using the Kaplan-Meier method and all point estimates include $95 \%$ confidence interval (CI). Statistical significance of the differences in Kaplan-Meier estimates was assessed using the log-rank test. As a level of statistical significance, $\alpha=0.05$ was used.

\section{Results}

There were 26 mCRC patients enrolled into the CORECT registry with well documented progression on cetuximab in combination with irinotecan-based chemotherapy (FOLFIRI 
or irinotecan alone) who received panitumumab monotherapy. Nineteen patients were male and 7 patients were female. The median age at cetuximab treatment initiation was 60 years (range=32-70). In most cases $(18 / 26)$, previous treatment with cetuximab was the second-line treatment, in 5 cases the thirdline therapy and only 3 patients were treated with cetuximab in the first-line treatment. After progression on cetuximab, all patients were treated with panitumumab monotherapy at standard dose of $6 \mathrm{mg} / \mathrm{kg}$ intravenously every 2 weeks. Partial response (PR) was achieved in $3(11.5 \%)$ patients, stable disease (SD) in 7 (26.9\%) patients, progressive disease (PD) in $13(50.0 \%)$ patients and, in $3(11.5 \%)$ cases, response was not evaluable (Figure 1A). Median progression-free survival (mPFS) from panitumumab treatment initiation was 2.8 months (95\% CI=1.5-4.0) (Figure 1B). Panitumumab was well tolerated. Twenty-two patients (86\%) had grade 1-2 dry skin or rash. Three patients had treatment-related grade 3-4 toxicities (1x skin toxicity $\mathrm{G} 4,1 \mathrm{x}$ skin toxicity $\mathrm{G} 3,1 \mathrm{x}$ pulmonary embolism G3 and 1x deep vein thrombosis G3). We did not find any relationship between previous therapy response and panitumumab treatment response or PFS with panitumumab and between cetuximab PFS and PFS with panitumumab (Figure 2A-C). We demonstrated, however, a significant association of miR-31-5p expression levels and PFS with cetuximab (median PFS of 12.3 vs. 4.9 months; $\mathrm{p}=0.038$, Figure $3 \mathrm{~A}$ ) but did not find any connection between expression level of miR-31-5p and PFS with panitumumab (Figure 3B).

\section{Discussion}

Just a few studies have only been published focusing on the safety and clinical efficacy of panitumumab following disease progression with cetuximab therapy (5-8). Metges et al. (PANERB trial) prospectively treated 32 wild-type KRAS mCRC patients with cetuximab and irinotecan followed by panitumumab monotherapy after progression. Remarkably, the Authors reported an objective response rate of $22 \%$ to panitumumab, including a disease control rate (objective response plus SD) of $73 \%$ in 11 patients who had previously responded to cetuximab and irinotecan (5). Similarly, of the 11 evaluable patients, Saif et al. noted minor radiographic responses in $3(27 \%)$ patients and SD in $3(27 \%)$ other patients after 8 weeks of therapy. Five (46\%) patients had evidence of PD and further therapy was stopped. The median duration of SD was 4 months. Among the 11 evaluable patients, 1 patient achieved $>50 \%$ reduction in carcinoembryonic antigen (CEA), 3 patients had a $25 \%$ reduction and 1 patient had minor reduction in CEA (6). When compared to these studies, in our case series of $26 \mathrm{mCRC}$ patients, we observed lower disease control rate being $38.5 \%$ with partial regression in $11.5 \%$ patients and disease stabilization in $27 \%$ patients with a median PFS of 3.42 months. In contrast, Wadlow et al. found no

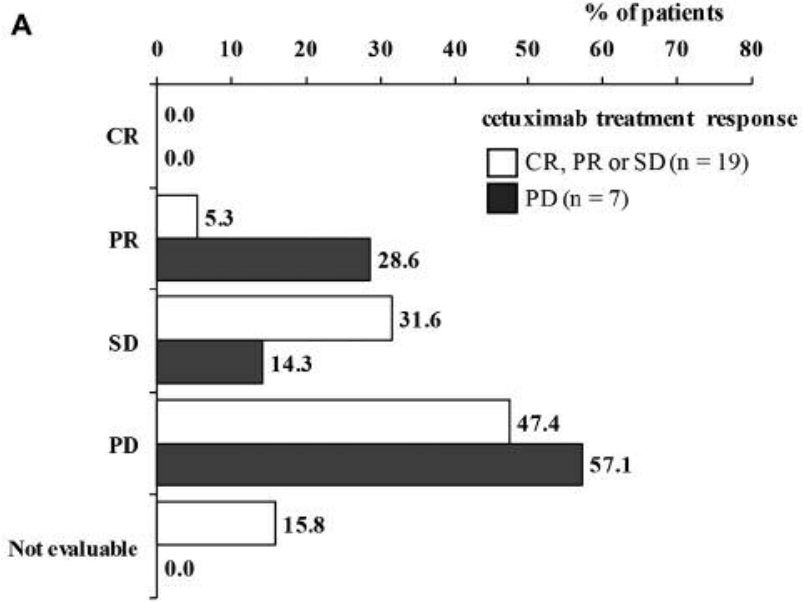

B

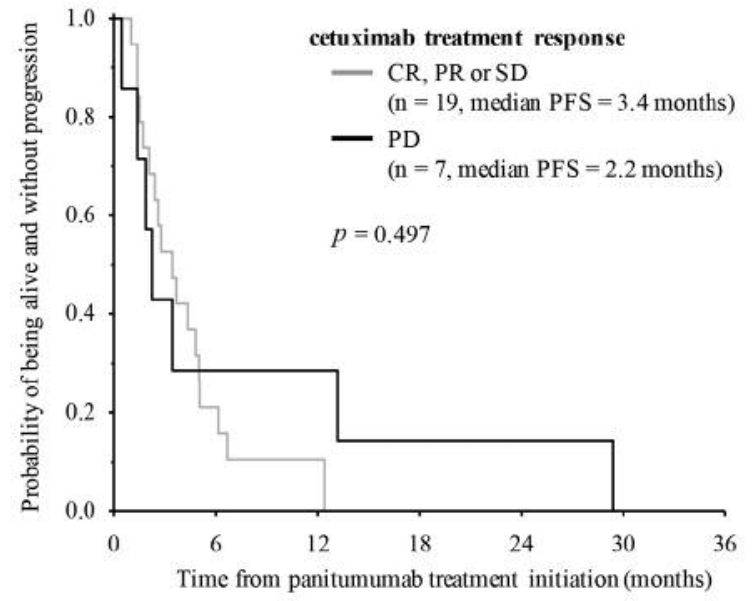

C

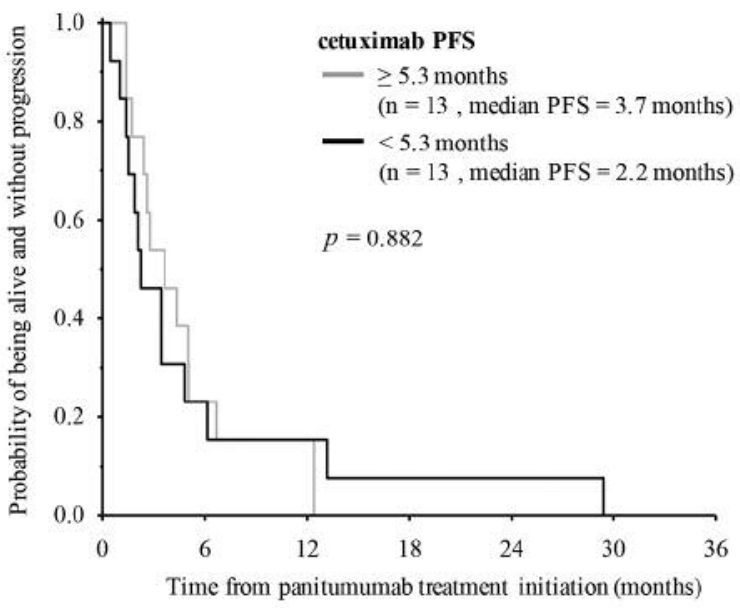

Figure 2. Relationship of cetuximab and consequent panitumumab therapeutic response and progression-free survival (PFS). Response to panitumumab in patients stratified according to their response to cetuximab (A). PFS with panitumumab treatment according to cetuximab treatment response $(B)$. PFS with panitumumab according to median PFS on cetuximab treatment $(C)$. PR, Partial response; $C R$, complete response; $S D$, stable disease; $P D$, progressive disease. 

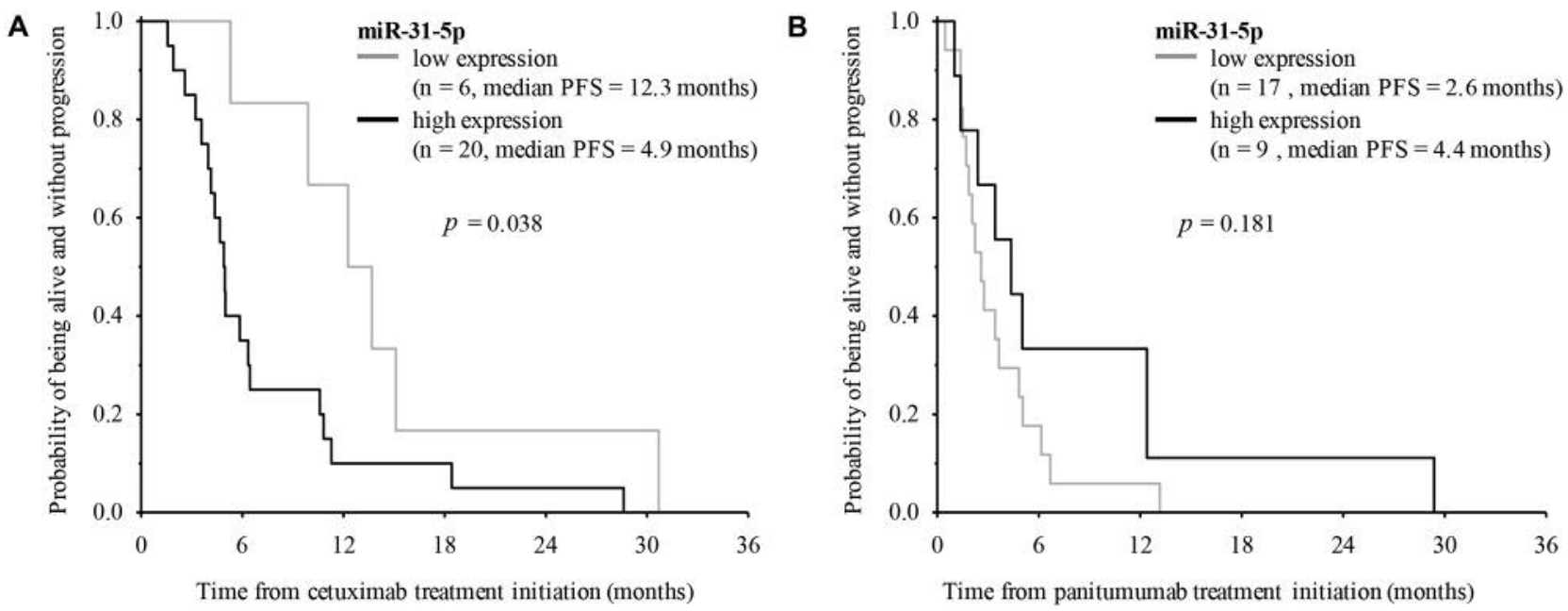

Figure 3. MiR-31-5p as predictive biomarker to anti-epidermal growth factor receptor (EGFR) therapy. Progression-free survival (PFS) from cetuximab treatment initiation according to miR-31-5p expression levels (A). PFS from panitumumab treatment initiation according to miR-31-5p expression level $(B)$.

responders and a SD rate of $45 \%$ with a median duration of only 1.7 months in a trial of $20 \mathrm{mCRC}$ patients (8). In all studies, including ours, panitumumab was well tolerated.

Currently, it is not clear why patients who had DP with cetuximab were able to derive clinical benefits from panitumumab. Recently, Montagut et al. revealed that the presence of the acquired EGFR ectodomain mutation (S492R) may provide a molecular explanation for the clinical benefits of panitumumab therapy in a subset of patients with mCRC who did not respond to cetuximab treatment (13). Another possibility is that the two antibodies may inhibit EGFR signaling via separate mechanisms and, as a result, it is also conceivable that distinct mechanisms of resistance may develop to the respective anti-EGFR antibodies (7).

We have not observed any link between response to cetuximab and consequent panitumumab treatment. We have further evaluated association of the newly described biomarker miR-31-5p and PFS with cetuximab and, consequently, with panitumumab treatment. We have confirmed our previous observations that higher expression levels of miR-31-5p in tumor tissue are significantly associated to shorter PFS with cetuximab; however, we did not observe any association between miR-31-5p levels and therapeutic response to panitumumab in $\mathrm{mCRC}$ patients after progression on cetuximab. Since panitumumab is a fully human monoclonal antibody ( $\operatorname{IgG} 2)$ and characterized by different immune responses in comparison to antibodydependent cellular cytotoxicity induced by the chimeric monoclonal antibody cetuximab (IgG1), we hypothesize that the specific predictive value of miR-31-5p in cetuximab therapy is associated with specific immune response induced by cetuximab but not panitumumab (12).
In conclusion, our relatively small case series suggests that it remains possible that a small subset of mCRC patients may benefit from panitumumab after progression on cetuximab. However, based on previous response to cetuximab and predictive biomarker miR-31-5p, this subset of patients can not be identified. This therapeutic approach should not be adopted until predictive biomarkers for panitumumab response in this setting have been discovered and validated.

\section{Acknowledgements}

The Authors would like to thank the following heads of the comprehensive cancer centers for their permission to use data of patients from their respective regional networks in CORECT registry: Dr. Vaclav Janovsky, Ceske Budejovice; Prof. Jindrich Finek, Plzen; Prof. Rostislav Vyzula, Brno; Dr Lubomir Slavicek, Jihlava, Prof. Renata Soumarova, Novy Jicin; Dr Jiri Bartos, Liberec; Prof. David Feltl, Ostrava; Prof. Jana Prausova, Prague; Prof. Jitka Abrahamová, Prague; Dr. Martina Kubecova, Prague; Martina Chodacka, Usti nad Labem; Prof. Jiri Petera, Hradec Kralove; Dr. Milan Kohoutek, Zlin; Prof. Bohuslav Melichar, Olomouc. We are obliged also to all physicians who provided data for the CORECT registry.

The maintenance of the CORECT registry is partly funded by Roche, Merck and Amgen. This work has been financially supported by the Czech Ministry of Health, grant nr. 16-31765A, project MZ CR - RVO (MOU, 00209805), by the Ministry of Education, Youth and Sports of the Czech Republic under the project CEITEC 2020 (LQ1601) and by a grant from the Czech Ministry of Education, Youth and Sports (NPU LO 1304), Czech Technological Agency: project TACR TE02000058.

\section{Disclosure Statement}

Dr. Kiss has received speaker's honoraria from Roche, Merck and Amgen. All other Authors state that they have no conflict of interest. 


\section{Informed Consent}

Patients were identified from a prospective institutional database and the study was approved by the local Research Ethics Committee.

\section{References}

1 Pietrantonio F, Cremolini C, Petrelli F, Di Bartolomeo M, Loupakis F, Maggi C, Antoniotti C, de Braud F, Falcone A and Iacovelli RL: First-line anti-EGFR monoclonal antibodies in panRAS wild-type metastatic colorectal cancer: A systematic review and meta-analysis. Crit Rev Oncol Hematol 96: 156-166, 2015.

2 Hagan S, Orr MC and Doyle B: Targeted therapies in colorectal cancer-an integrative view by PPPM. EPMA J 4: 3, 2013.

3 Heinemann V, von Weikersthal LF, Decker T, Kiani A, VehlingKaiser U, Al-Batran SE, Heintges T, Lerchenmüller C, Kahl C, Seipelt G, Kullmann F, Stauch M, Scheithauer W, Hielscher J, Scholz M, Müller S, Link H, Niederle N, Rost A, Höffkes HG, Moehler M, Lindig RU, Modest DP, Rossius L, Kirchner T, Jung A and Stintzing S: FOLFIRI plus cetuximab versus FOLFIRI plus bevacizumab as first-line treatment for patients with metastatic colorectal cancer (FIRE-3): A randomised, open-label, phase 3 trial. Lancet Oncol 15: 1065-1075, 2014.

4 Modest DP, Stintzing S, von Weikersthal LF, Decker T, Kiani A, Vehling-Kaiser U, Al Batran SE, Heintges T, Lerchenmüller C, Kahl C, Seipelt G, Kullmann F, Stauch M, Scheithauer W, Held S, Möhler M, Jung A, Kirchner T and Heinemann V: Impact of Subsequent Therapies on Outcome of the FIRE-3/AIO KRK0306 Trial: First-Line Therapy With FOLFIRI Plus Cetuximab or Bevacizumab in Patients With KRAS Wild-Type Tumors in Metastatic Colorectal Cancer. J Clin Oncol 33: 37183726, 2015

5 Metges J, Raoul J, Achour N, Capitain O, Gourlaouen A, Ramée A, Egreteau J, Douillard J, Traoré S and Grudé F: PANERB study: Panitumumab after cetuximab-based regimen failure. J Clin Oncol 28: suppl; abstr: e14000, 2010.

6 Saif MW, Kaley K, Chu E and Copur MS: Safety and efficacy of panitumumab therapy after progression with cetuximab: Experience at two institutions. Clin Colorectal Cancer 9: 315318,2010

7 Sonoda H, Mekata E, Shimizu T, Endo Y and Tani T: Safety and efficacy of panitumumab therapy after metastatic colorectal cancer progression with cetuximab: Experience at a single Japanese institution. Oncol Lett 5: 1331-1334, 2013.
8 Wadlow RC, Hezel AF, Abrams TA, Blaszkowsky LS, Fuchs CS, Kulke MH, Kwak EL, Meyerhardt JA, Ryan DP, Szymonifka J, Wolpin BM, Zhu AX and Clark JW: Panitumumab in patients with KRAS wild-type colorectal cancer after progression on cetuximab. Oncologist 17: 14, 2012.

9 Nemecek R, Tomasek J, Hejduk K and Bortlicek Z: CORECT registry. Klin Onkol 25: 480-485, 2012.

10 Manceau G, Imbeaud S, Thiébaut R, Liébaert F, Fontaine K, Rousseau F, Génin B, Le Corre D, Didelot A, Vincent M, Bachet JB, Chibaudel B, Bouché O, Landi B, Bibeau F, Leroy K, Penault Llorca F, Van Laethem JL, Demetter P, Tejpar S, Rossi S, Mosakhani N, Osterlund P, Ristamäki R, Sarhadi V, Knuutila $\mathrm{S}$, Boige V, André $\mathrm{T}$ and Laurent-Puig P: Hsa-miR-31-3p expression is linked to progression-free survival in patients with KRAS wild-type metastatic colorectal cancer treated with antiEGFR therapy. Clin Cancer Res 20: 3338-3347, 2014.

11 Igarashi H, Kurihara H, Mitsuhashi K, Ito M, Okuda H, Kanno S, Naito T, Yoshii S, Takahashi H, Kusumi T, Hasegawa T, Sukawa Y, Adachi Y, Okita K, Hirata K, Imamura Y, Baba Y, Imai K, Suzuki H, Yamamoto H, Nosho K and Shinomura Y: Association of microrna-31-5p with clinical efficacy of antiEGFR therapy in patients with metastatic colorectal cancer. Ann Surg Oncol 22: 2640-2648, 2015.

12 Mlcochova J, Faltejskova-Vychytilova P, Ferracin M, Zagatti B, Radova L, Svoboda M, Nemecek R, John S, Kiss I, Vyzula R, Negrini $M$, and Slaby O: MicroRNA expression profiling identifies $\operatorname{miR}-31-5 p / 3 p$ as associated with time to progression in wild-type RAS metastatic colorectal cancer treated with cetuximab. Oncotarget 6: 38695-38704, 2015.

13 Montagut C, Dalmases A, Bellosillo B, Crespo M, Pairet S, Iglesias M, Salido M, Gallen M, Marsters S, Tsai SP, Minoche A, Seshagiri S, Serrano S, Himmelbauer H, Bellmunt J, Rovira A, Settleman J, Bosch F and Albanell J: Identification of a mutation in the extracellular domain of the Epidermal Growth Factor Receptor conferring cetuximab resistance in colorectal cancer. Nat Med 18: 221-223, 2012.
Received July 12, 2016

Revised July 19, 2016

Accepted July 20, 2016 